\title{
ARTICLE \\ Epigallocatechin-3-gallate local pre-exposure application prevents SHIV rectal infection of macaques
}

\author{
J. B. Liu' ${ }^{1}$, J. L. Li ${ }^{2}$, K. Zhuang ${ }^{1}$, H. Liu' ${ }^{1}$, X. Wang ${ }^{2}$, Q. H. Xiao' ${ }^{1}$ X. D. Li' ${ }^{1}$, R. H. Zhou ${ }^{1}$, L. Zhou ${ }^{1}$, T. C. Ma ${ }^{1}$, W. Zhou ${ }^{3}$, M. Q. Liu ${ }^{3}$ and W. Z. Ho ${ }^{1,2}$
}

\begin{abstract}
Epigallocatechin-3-gallate (EGCG), a natural and major ingredient of green tea, has been shown to have anti-inflammation and antiHIV-1 properties. We demonstrated that the intrarectal administration of EGCG could protect rhesus macaques from repetitive,

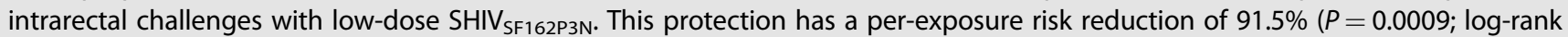
test) and a complete protection of $87.5 \%(P<0.001$; Fisher's exact test). All protected animals showed no evidence of systemic and mucosal SHIV infection as demonstrated by the absence of viral RNA, DNA and antibodies. In contrast, all controls became infected after repeated SHIV challenges (a median of 2.5 times, range of 1-8 times). Mechanistically, EGCG could block the binding of HIV-1 gp120 to CD4 receptor and suppress the macrophage infiltration/activation in the rectal mucosa of macaques. These data support further clinical evaluation and development of EGCG as a novel, safe and cost-effective microbicide for preventing sexual transmission of HIV-1.
\end{abstract}

Mucosal Immunology (2018) 11:1230-1238; https://doi.org/10.1038/s41385-018-0025-4

\section{INTRODUCTION}

In the absence of a protective vaccine or a cure for HIV-1 infection, intervention with highly potent and cost-effective inhibitors that target at the initial sites of HIV-1 infection is an ideal strategy for slowing the AIDS pandemic. Currently, HIV-1 infection spreads predominantly through sexual transmission, in which the virus gains access to a new host through the penile, rectal or vaginal mucosal tissues. During sexual intercourse, HIV-1-infected men transmit the virus through their semen, which carries free-floating virus, as well as the infected cells. ${ }^{1}$ Semen contains amyloid fibrils that potently enhance HIV-1 or SIV/SHIV infectivity in vitro. ${ }^{2-4}$ Therefore, to inhibit HIV-1 during sexual intercourse represents the most effective way in preventing transmission of the virus.

Microbicides targeting the early events in sexual transmission of HIV-1 have been extensively studied. ${ }^{5-8}$ Several recent studies reported that pre-exposure prophylaxis with the antiretrovirals (ARVs) was effective in the prevention of SHIV transmission in nonhuman primate (NHP) model., ${ }^{9,10}$ These ARVs-containing microbicides were usually used in the formulation of topical gels. ${ }^{7}$ However, the topical gel microbicides are associated with rectal mucosal damage, manifested as histological abnormality, and induction of proinflammatory response. ${ }^{11}$ In addition, ARVs-based microbicides are not effective on resistant HIV-1 strains and can induce viral resistance. Furthermore, semen can impair the antiviral efficacy of ARVs that act on intracellular targets at different steps of viral replication cycle. ${ }^{4}$ As compared with ARVs or other chemical drugs, the use of natural products for prevention of HIV-1 mucosal transmission might have some advantages, as they have low toxicity, minor side-effects, and are cost-effective.
The consumption of green tea has many physiological and pharmacological health benefits. Mounting evidence has shown that epigallocatechin-3-gallate (EGCG), a natural and major ingredient of green tea, not only has anti-inflammatory and antioxidative effects, but also possesses antiviral abilities against diverse families of viruses, including HIV-1. ${ }^{12}$ In vitro studies have demonstrated that EGCG at a physiologic concentration could inhibit HIV-1 infection/replication in peripheral blood mononuclear cells (PBMCs) and macrophages. ${ }^{13}$ In addition, EGCG could remodel seminal amyloid fibrils ${ }^{14}$ and effectively suppress semen-mediated enhancement of HIV-1 replication. ${ }^{15}$ EGCG can interfere with several aspects of the HIV-1 life cycle, including the destruction of virions, ${ }^{16,17}$ the inhibition of reverse transcriptase and integrase activities of the virus. ${ }^{18,19}$ Most conclusively, several independent studies have confirmed that EGCG can bind to the same molecular pocket on CD4 receptor as does HIV-1 gp120, thus preventing the initial attachment of the viruses to $\mathrm{CD}^{+}$ $\mathrm{T}$ cells. $^{20,21}$ We recently reported that EGCG could also inhibit macaque SEVI (semen-derived enhancer of virus infection)mediated enhancement of SIV/SHIV infection and degrade the formation of macaque semen-derived amyloid fibrils. ${ }^{22} \mathrm{~A}$ most recent clinical study showed that EGCG is a well-tolerated and non-toxic therapeutic agent that can upregulate protective CC chemokines against HIV-1. ${ }^{23}$ Taken together, these observations strongly argue for further studies, particularly in vivo investigations, on the anti-HIV-1 effect of EGCG as an entry inhibitor. In the present study, we evaluated whether mucosal pre-exposure application of EGCG can prevent SHIV rectal infection of rhesus macaques.

${ }^{1}$ Animal Biosafety Level III Laboratory at Center for Animal Experiment, Wuhan University School of Basic Medical Sciences, Wuhan, Hubei 430071, China; ${ }^{2}$ Department of Pathology and Laboratory Medicine, Temple University Lewis Katz School of Medicine, Philadelphia, PA 19140, USA and ${ }^{3}$ Department of Virology, Wuhan Centers for Disease Prevention and Control, Wuhan, Hubei 430015, China

Correspondence: W Z. Ho (wenzheho@temple.edu)

These authors contributed equally: J.B. Liu, J.L. Li.

Received: 6 December 2017 Revised: 23 February 2018 Accepted: 27 March 2018

Published online: 31 May 2018 
a
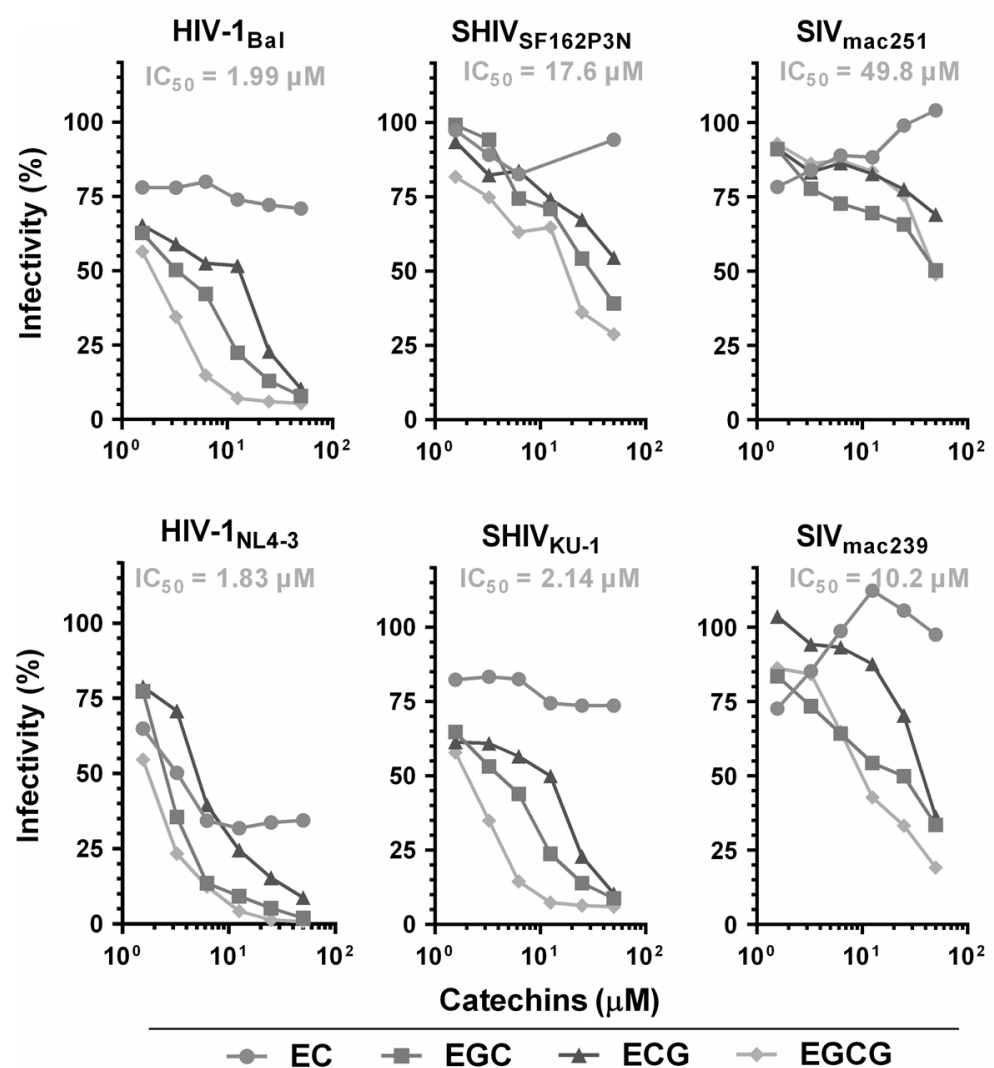

b
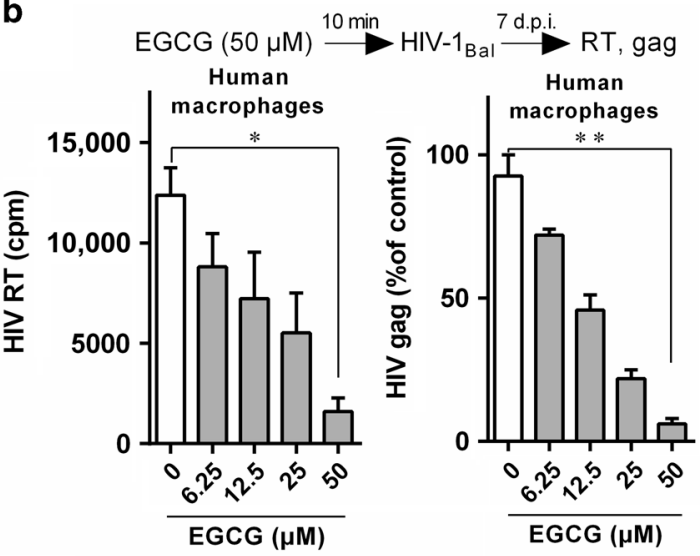

C

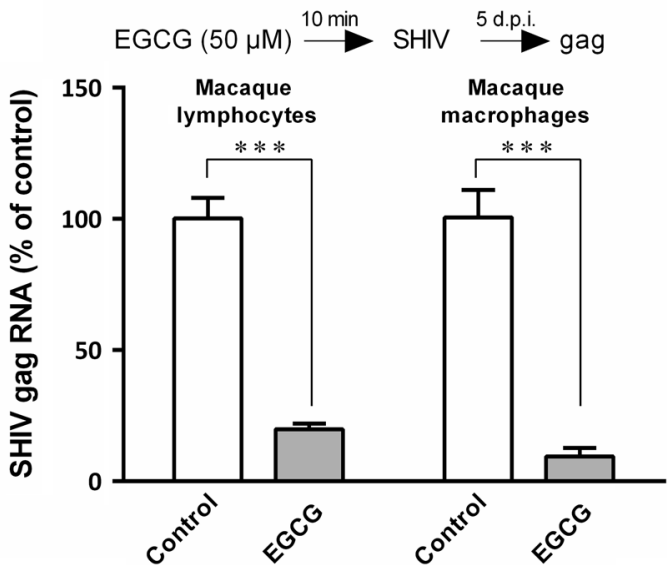

Fig. 1 EGCG inhibits viral infectivity of a broad spectrum of AIDS-related viruses. a TZM-bl cells were treated with the indicated concentrations of green tea-derived catechins (EC, EGC, ECG, and EGCG) for $10 \mathrm{~min}$ prior to infection with different strains of HIV-1 (Bal, NL4-3), SHIV (SF162P3N, KU-1), or SIV (mac239, mac251). Viral infectivity was assessed by luciferase activity, which is expressed as a percentage relative to that of the control (untreated). The half maximal inhibitory concentration (IC $\left.C_{50}\right)$ of EGCG is indicated, which was calculated based on the untreated control by the method of Reed and Muench. b Human peripheral blood monocyte-derived macrophages were incubated with the

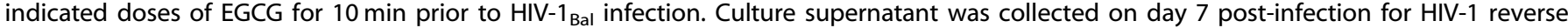
transcriptase (RT) assay. Cellular RNA was subjected to the real time RT-PCR for HIV-1 gag and GAPDH RNA. Data are expressed as HIV-1 RNA levels relative (\%) to untreated control, which is defined as $100 \%$. c Primary lymphocytes and macrophages from rhesus macaques were treated with or without EGCG $(50 \mu \mathrm{M})$ for 10 min prior to SHIV $\mathrm{SF}_{162 \mathrm{P} 3 \mathrm{~N}}$ infection. Intracellular gag RNA was measured by the real time PCR at day 5 post-infection. Data are shown as mean $\pm S D$, representative of three independent experiments with $3-4$ replicates. ${ }^{*} P<0.05$, ${ }^{* *} P<0.01$ and ${ }^{* * *} P<0.001$

\section{RESULTS}

EGCG inhibits HIV/SIV/SHIV in vitro

The major active ingredients of green tea are polyphenolic compounds, known as catechins, which include EGCG, EGC, ECG, and EC. EGCG accounts for $50 \%$ of the total green tea catechins. We first examined whether EGCG and other green tea catechins have the ability to inhibit infections with different strains of HIV-1, SHIV and SIV in TZM-bl cells. Among the green tea catechins, EGCG was the most potent inhibitor of the viruses (Fig. 1a). In addition, EGCG dose-dependently inhibited HIV-1 Bal $_{\text {replication in }}$ primary human macrophages (Fig. 1b). Furthermore, EGCG could inhibit SHIV infection of primary lymphocytes and macrophages of macaques (Fig. 1c). Evaluation of cytotoxicity revealed that the treatment of TZM-bl and PBMCs of macaques with the catechins for as long as $48 \mathrm{~h}$ at the dose as high as $100 \mu \mathrm{M}$ had little toxic effect (Supplementary Figures 1 and 2).

EGCG has little toxicity on rectal mucosa

To determine the in vivo protective effect of EGCG on SHIV rectal infection of macaques, we first assessed the toxicity of EGCG on rectal mucosa of macaques and mice. We demonstrated that the rectal application of $5 \mathrm{mM}$ EGCG for $10 \mathrm{~min}$ had little toxic effect on the rectal mucosa of macaques, as shown by the normal microstructures of epithelium, lamina propria, and intestinal glands in the rectum mucosa and submucosa (Supplementary Figure 3a) and lack of fecal blood (Supplementary Table 1). We also showed that EGCG had little effect on the expression of inflammatory cytokines and toxicity on the rectal mucosa of mice which is evidenced by normal intestinal microstructures, stool consistency, and lack of fecal blood (Supplementary Table 2 and Figure 3).

EGCG protects macaques from rectal SHIV infection

We next examined the protective effect of EGCG on repetitive and intra-rectal challenges with low-dose SHIV $_{\text {SF162P3N }}$ in macaques (Fig. 2a). The assessment of the plasma SHIV RNA over the course of the study showed that all animals ( 8 out of 8 ) in the control group were infected, which required a median of 2.5 times of challenges (range from 1 to 8 times, Fig. 2b). In contrast, only 1 out of 8 animals in the EGCG group became infected (Fig. 2c). Evaluation of SHIV RNA and proviral DNA in PBMCs and the biopsied specimens (rectum and inguinal lymph nodes, LNs) at week 20 postinfection revealed infection in control animals, while no evidence of infection in 7 out of 8 EGCG-treated animals (Supplementary Figure 4). 
a
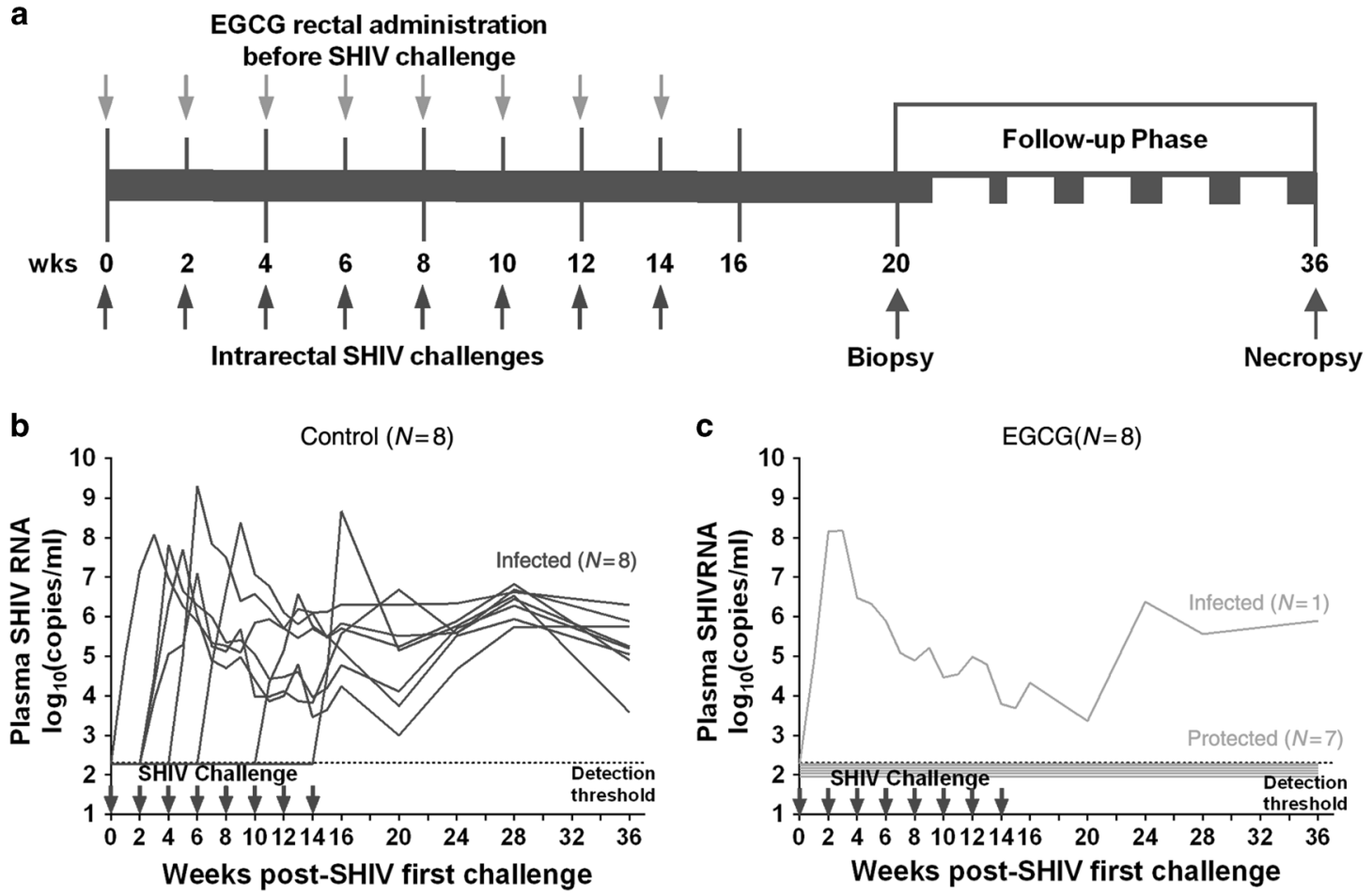

Fig. 2 EGCG protects macaques from intra-rectal SHIV infection. a Experimental design of EGCG protective effect on macaques. Sixteen male macaques were administered with $2 \mathrm{ml}$ of $5 \mathrm{mM}$ EGCG ( 8 animals) or $2 \mathrm{ml}$ of PBS (8 animals) atraumatically in rectum 10 min prior to each SHIV $_{\text {SF162P3N }}$ challenge. All animals were rectally inoculated with SHIV SF162P3N $_{10}$ TCID $\left._{50}\right)$ for up to 8 times or until infection occurred. All animals were biopsied at week 20 and necropsied at week 36 postinfection for the evaluation of SHIV RNA and proviral DNA in the multiple tissues. b, c Longitudinal assessment of the plasma SHIV RNA (copies $\mathrm{ml}^{-1}$ ) levels in the animals with intrarectal pretreatment with PBS (control) or EGCG prior to SHIV challenges (up to 8 times or till infection occurred). Duplicate plasma samples were analyzed for SHIV RNA detection. Animals were considered infected and the virus challenges were stopped following two consecutive positive plasma SHIV RNA results

Because gut-associated lymphoid tissue (GALT) is an important target organ of HIV-1 and may serve as a key viral reservoir, we also examined SHIV proviral DNA and RNA in the gastrointestinal tract (Gl) tissues, lymph nodes (LNs) and intraepithelial lymphocytes (IELs) of study animals necropsied at week 36 postinfection. As shown in Fig. 3 and Supplementary Table 3, SHIV RNA and proviral DNA were detected in all analyzed tissues (GI tissues, LNs and IELs) from control animals. In contrast, only one animal (WRE08) in EGCG group had detectable SHIV RNA and DNA in the GI tissues, LNs and IELs. In addition to the plasma viral loads, we also examined plasma antibody against SHIV in the study animals. Consistent with the viral load data, while all control animals were positive for SHIV-specific antibody, only one animal (WRE08) in EGCG group had detectable SHIV antibody (Supplementary Table 3). This animal had the highest CD69 and on CD4 ${ }^{+}$and $\mathrm{CD}^{+} \mathrm{T}$ cells in the first 2 weeks post-infection (Supplementary Figure 5). In addition, there was a significant decrease of $\mathrm{CD}^{+}{ }^{+}$ cell numbers in blood of this animal at week 3 after SHIV infection (Supplementary Figure 6).

Statistically, the EGCG treatment afforded a protective efficacy of $91.5 \%$ [1-(hazard ratio), $P=0.0009$, log-rank test] reduction in the per-exposure acquisition risk as compared with control (Fig. 4). The complete protection for macaques in the EGCG group was 87.5\% $(P<0.001$, Fisher's exact test).

Mechanisms of EGCG-mediated in vitro and in vivo anti-HIV activities

We first studied the mechanisms of the in vitro findings that the pre-treatment of the target cells (TZM-bl, primary macaque lymphocytes and macrophages) with EGCG potently inhibited HIV/SHIV/SIV infection. As shown in Fig. 5a, b, EGCG pretreatment of $\mathrm{T}$ cells could block the binding of CD4 antibody to CD4 receptor. We also observed that the incubation of $\mathrm{CD}^{+} \mathrm{T}$ cells with EGCG blocked the binding of FITC-conjugated HIV-1 gp120 to the cells (Fig. $5 c$ ). To determine in vivo mechanism of EGCGmediated protection of macaques from SHIV rectal infection, we examined the effect of EGCG on the infiltration and activation of $T$ cells and macrophages in rectal mucosa (Fig. 6a). EGCG rectal administration significantly reduced the infiltration of $\mathrm{CD}^{+} 8^{+}$ macrophages $(P<0.01)$, but not $\mathrm{CD}^{+} \mathrm{T}$ cells in rectal mucosa (Fig. $6 \mathrm{~b}, \mathrm{c})$. In addition, EGCG pretreatment suppressed the local immune activation as shown by the diminished expression of $\mathrm{CD} 163^{+}$cells $(P<0.05)$ and $\mathrm{HLA}^{-\mathrm{DR}^{+}}$cells $(P<0.01)$ in rectal mucosa (Fig. 6d, e).

\section{DISCUSSION}

Worldwide, sexual intercourse is currently the predominant mode of HIV-1 transmission, in which unprotected anal sex is riskier than unprotected vaginal sex. ${ }^{24}$ The rectum is a particularly susceptible entry site for HIV-1 or SHIV infection, as it is composed of only a single layer of columnar epithelium. In addition, the rectal mucosa contains numerous CD4-bearing target cells $\left(\mathrm{CD}^{+}{ }^{+} \mathrm{T}\right.$ cells, macrophages and DCs). ${ }^{25}$ The risk of HIV transmission during anal intercourse is estimated to be around 18 times greater than that in vaginal intercourse, according to the results of a metaanalysis. ${ }^{26}$ Therefore, the interventions to target the rectal site are critical in preventing sexual transmission of HIV-1. In this study, we demonstrate for the first time that EGCG can protect 7 out of 8 macaques from repeated intra-rectal challenges with SHIV, resulting in a per-exposure risk reduction of $91.5 \%(P=0.0009$ by the log-rank test) and a complete protection of $87.5 \%(P<$ 
a
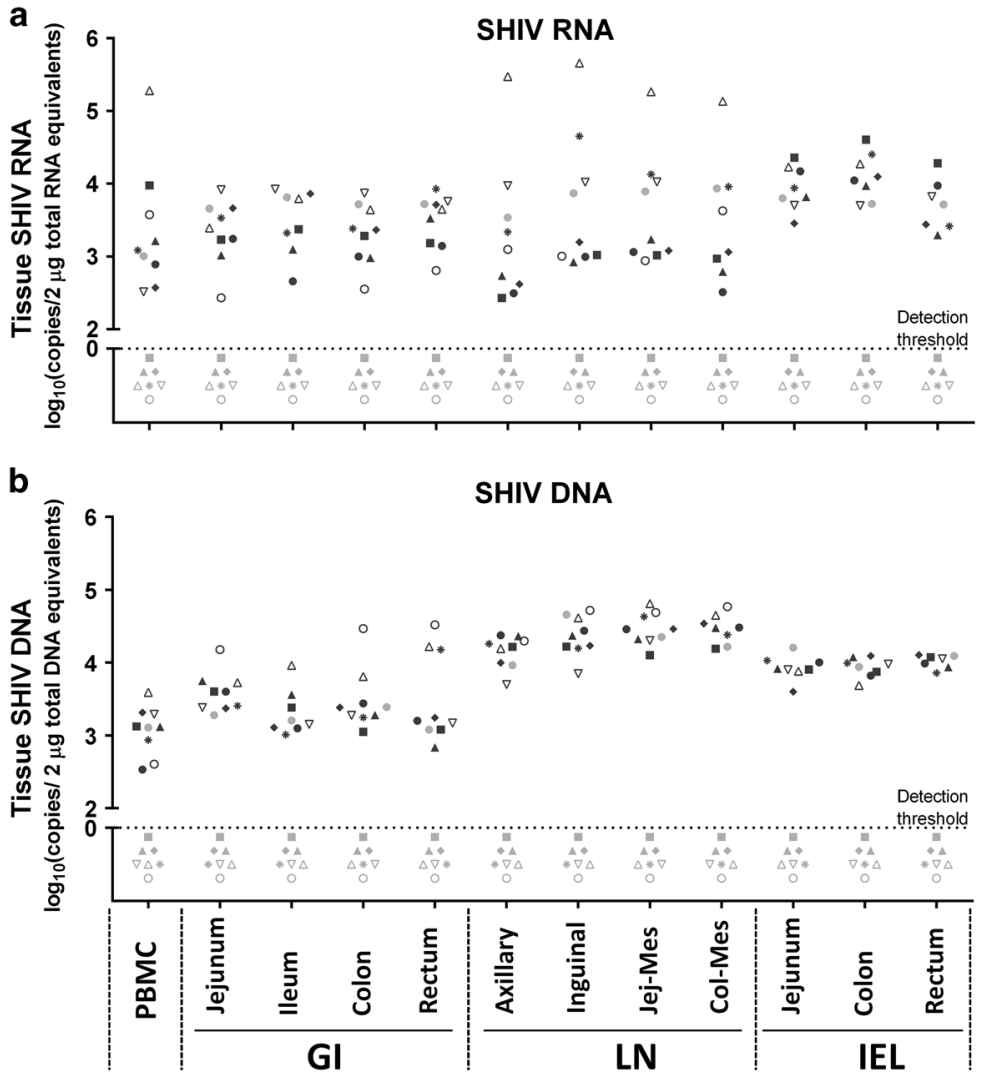

SHIV RNA

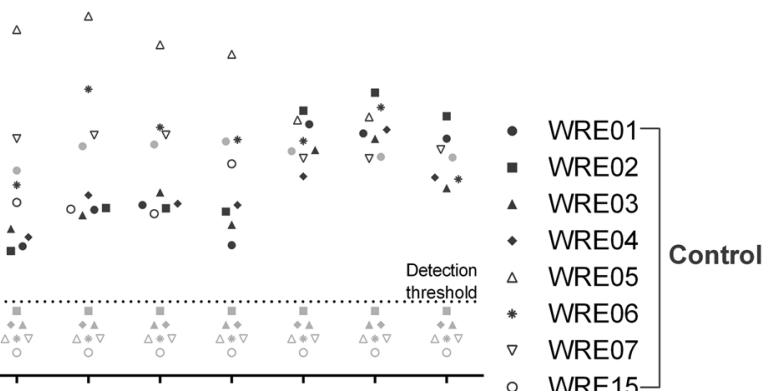

WRE08

WRE09

WRE10

WRE11

WRE12

WRE13

WRE14

WRE16

Fig. 3 SHIV RNA and DNA detection in multiple tissues necropsied at week 36 post first SHIV challenge. SHIV RNA (a) and DNA (b) assays in the indicated tissues from the animals of PBS control (red symbols, $n=6-8$ ) and EGCG group (green symbols, $n=8$ ) at necropsy. Log 10 SHIV copies $2 \mu \mathrm{g}^{-1}$ total genomic RNA or DNA equivalents are shown. Symbols represent individual animals and are pooled from three independent experiments. Triplication tissue samples were conducted in each independent experiment. The dot line: the detection threshold. Gl gastrointestinal tract, LN lymph nodes, Jej-Mes jejunal mesenteric, Col-Mes colonal mesenteric, IEL intraepithelial lymphocytes

0.001 by Fisher's exact test). All protected animals showed no evidence of systemic and mucosal SHIV infection as evidenced by the absence of viral RNA, DNA and antibodies. Interestingly, EGCG failed to protect one animal from SHIV infection. This animal appeared to be highly susceptible to SHIV challenge, as it became infected after only one-time of exposure to the virus. In contrast, all control animals except one required 2 or more repeated SHIV challenges (Fig. 2b, c). In examining the immune activation markers (CD69 and HLA-DR) on $\mathrm{CD}^{+}$and $\mathrm{CD}^{+}{ }^{+}$T cells of all infected animals, we found that this unprotected animal had the highest $\mathrm{CD} 69$ expression on $\mathrm{CD}^{+}$and $\mathrm{CD}^{+} \mathrm{T}$ cells among all infected monkeys in the first 2 weeks post-infection (Supplementary Figure 5).

EGCG has been reported to have in vitro antiviral effect on different subtypes, strains, and clinical isolates of HIV-1 in primary human $\mathrm{CD}^{+}{ }^{+} \mathrm{T}$ cells and macrophages. ${ }^{13}$ We showed that EGCG had broad inhibitory effect on different strains of HIV-1, SIV, and SHIV in TZM-bl cells, macaque lymphocytes and macrophages (Fig. 1). Studies from different laboratories have demonstrated that EGCG exhibits antiviral activity at multiple steps of HIV-1 infection/replication cycle, including the inhibition of HIV-1 reverse transcriptase, ${ }^{18}$ protease, $^{16}$ and proviral genome integration. ${ }^{19}$ Importantly, EGCG-mediated HIV-1 inhibition appears to be its interference with HIV-1 entry into the target cells. EGCG can directly disassemble HIV-1 virion, ${ }^{16,17}$ block HIV-1 gp120 binding to CD4 molecule on T cells, ${ }^{20,21,27}$ and degrade the seminal amyloid fibrils (a semen-derived enhancer of virus infection, SEVI) that reportedly enhances HIV-1 infection/transmission. ${ }^{2,4,14,28}$ We found that EGCG specifically reduced the surface expression of CD4 receptor, but not CD3 receptor on human T cells (Fig. 5a), suggesting that EGCG blocks the binding of CD4 antibody to CD4 receptor (Fig. 5b). Also we showed that EGCG could block the binding of FITC-conjugated HIV-1 gp120 to CD4 receptor (Fig. 5c). More importantly, we demonstrated that in vivo application of EGCG could suppress the macrophage infiltration/activation in the rectal mucosa of macaques. The protective effect of EGCG was also observed in a recent human study, showing that EGCG oral administration upregulated the CC chemokines (MIP- $1 \alpha / \beta$ and RANTES) against HIV-1. ${ }^{23}$ These combined in vitro and in vivo observations provide a possible mechanism for EGCG-mediated rectal protection from SHIV infection.

These combined anti-HIV-1 actions of EGCG should reduce the risk of developing emergence of resistant viruses during the course of treatment. Furthermore, EGCG may confer an additional advantage over ARVs as it can counteract semen-mediated enhancement of HIV-1 infection. ${ }^{22,28}$ We recently reported that EGCG counteracted macaque SEVI-mediated enhancement of SIV/ SHIV infection of macaque PBMCs. ${ }^{22}$ This finding is clinically relevant and important, as it has been reported that the anti-HIV-1 activity of a variety of potential microbicides could be compromised by the presence of semen or SEVI amyloid fibrils. ${ }^{4}$ Therefore, in order to identify the best candidates for the prevention of HIV-1 sexual transmission, it is necessary to evaluate the anti-HIV-1 efficacy of the ARVs-formulated microbicides in the presence of semen. ${ }^{29-31}$

EGCG is generally considered a natural product with minimal toxicity. Clinically, EGCG has been found to be safe and well tolerated by the study subjects and the reported adverse events were rated as mild events. ${ }^{32,33}$ EGCG administration is safe in healthy individuals, at oral dose of $800 \mathrm{mg}$ per day over a time 


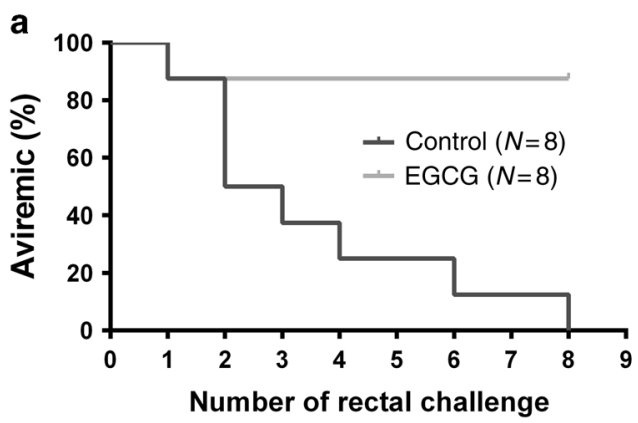

b

\begin{tabular}{|l|l|l|l|l|l|}
\hline & $\begin{array}{l}\text { Hazard } \\
\text { ratio } \\
(\mathbf{9 5 \%} \mathrm{Cl})\end{array}$ & $\begin{array}{l}\text { Per-exposure } \\
\text { risk } \\
\text { reduction }\end{array}$ & $\begin{array}{l}P \text {-value } \\
\text { vs } \\
\text { control }^{\#}\end{array}$ & $\begin{array}{l}\text { Complete } \\
\text { protection }\end{array}$ & $\begin{array}{l}P \text {-value vs } \\
\text { control }^{\$}\end{array}$ \\
\hline EGCG & $\begin{array}{l}0.080 \\
(0.022-0.326)\end{array}$ & $91.5 \%$ & 0.0009 & $87.5 \%$ & 0.001 \\
\hline Control & 1 & N/A & N/A & $0 \%$ & N/A \\
\hline & "Log-rank (Mantel-Cox) test & FFisher's exact test \\
\hline
\end{tabular}

Fig. 4 Protective efficacy of EGCG local pre-exposure application on SHIV rectal transmission in macaques. a Kaplan-Meier plot demonstrating the protection in EGCG-treated animals $(n=8)$ relative to PBS-treated animals $(n=8)$. b Statistical analyses include the hazard ratio with $95 \%$ confidence interval and the per-exposure reduction of acquisition risk in each group, with $P$ values reflecting log-rank test and Fisher's exact test

period of 4 weeks, which is equivalent to about 8-16 cups of green tea a day. ${ }^{34}$ Ullman et al. ${ }^{35}$ reported that purified EGCG at the doses up to $1600 \mathrm{mg}$ per day were generally well-tolerated and safe to use clinically. Oral application of EGCG in mouthwash formulation at the dose of $35-87 \mathrm{mM}$ for $2 \mathrm{~min}$ daily for 7 days had little systemic side effects. ${ }^{36} \mathrm{~A}$ recent phase I clinical trial showed that the drug formulation of EGCG, Polyphenon $E$, is a well-tolerated, non-toxic therapeutic agent with no significant adverse effects at up to $1600 \mathrm{mg}$ per day for 14 days. ${ }^{23}$ Our evaluation of cytotoxicity in the cell cultures showed that EGCG at doses up to $50 \mu \mathrm{M}$ had little cytotoxicity to TZM-bl cells and the primary macaque PBMCs. It was reported that the viability of HIVinfected human lymphocytes and macrophages was not significantly inhibited by EGCG over a range of $1-100 \mu \mathrm{M} .^{13}$ As compared to the concentrations of EGCG used in the cell cultures, higher dose $(5 \mathrm{mM})$ of EGCG was administered in the animal experiments. The rationale for use of this EGCG dose was based on the consideration that the macaque rectum contains multiple factors (feces, mucus, a number of microbial flora and metabolites) that could dilute and interfere with EGCG, reducing its antiviral activity. Nevertheless, we showed that $5 \mathrm{mM}$ EGCG had little toxicity and inflammatory effects on rectal mucosa of macaques and mice, as evidenced by the normal rectal epithelium structure and lack of inflammatory cytokine induction after the EGCG application (Supplementary Figure 3, Tables 1 and 2). The dosage of the FDA approved green tea extract Polyphenon E ointment (Veregen) for topical treatment of genital warts is about 100-300 $\mathrm{mM}^{37-39}$

Because sexual intercourse is a primary route of HIV-1 transmission worldwide and vaginal mucosal surface is a major site of the heterosexual transmission, future studies to determine the protective effect of EGCG on SHIV vaginal infection are also necessary. By extrapolating, it is likely that the same benefits of EGCG shown in the SHIV rectal infection model might apply to the prevention of vaginal SHIV infection. This supposition, however, will need to be confirmed experimentally given the differences between the rectal and vaginal transmission of HIV $-1 .{ }^{25}$ The fact that EGCG is very stable in acidic solution, ${ }^{40}$ a condition similar to vaginal environment $(\mathrm{pH}$ range of 3.8-4.5), supports the future studies on use of EGCG for prevention of intravaginal HIV-1 transmission. Importantly, it is necessary to develop EGCG formulations that have stable, effective and long-lasting action on inactivation of HIV-1 in vaginal lumen and rectum. For example, EGCG in a buffered gel or film should be able to maintain an acidic $\mathrm{pH}$ in the presence of semen.

Altogether with the known low toxicity, anti-inflammation and anti-viral properties of EGCG, our results argue in favor for more in vivo studies to examine the persistence, metabolism and chemical stability of EGCG at the mucosal exposure sites. These investigations are crucial for further clinical evaluation and development of EGCG as a safe, cost-effective and naturopathic microbicide for preventing sexual transmission of HIV-1, particularly in resource-poor settings.

\section{METHODS}

\section{Animals}

Sixteen adult male rhesus macaques were used to examine the protective effect of EGCG on prevention of SHIV rectal infection. The animals were obtained from the Kunming Institute of Zoology of Chinese Academy of Science. The animals were individually housed at the Animal Biosafety Level-III Laboratory at the Center for Animal Experiment of Wuhan University with AAALAC International accreditation (001274). All the animal protocols were approved by the Institutional Animal Care and Use Committee (approval number: 2015024) of Wuhan University in accordance with the NIH "Guide for the Care and Use of Laboratory Animals". All the details of animal welfare were carried out in accordance with the recommendations of the Weatherall report, "The Use of Nonhuman Primates in Research". All the animals in this study were experimentally naive at the beginning and negative for antibodies against the specific pathogens. All the procedures were performed under anesthesia with ketamine, and necessary efforts were taken to minimize suffering, improve housing conditions, and provide enrichment opportunities for the study animals.

\section{Cells and viruses}

TZM-bl cells with integrated firefly luciferase gene under the control of HIV-1 LTR were obtained from the NIH AIDS Research Program and grown in DMEM medium. Human and macaque PBMCs were prepared by Ficoll gradient centrifugation and cultured in RPMI medium containing $10 \% \mathrm{FBS}, 100 \mathrm{U} \mathrm{ml}^{-1}$ penicillin, $0.1 \mathrm{mg} \mathrm{ml}^{-1}$ streptomycin, $2 \mathrm{mM}$ L-glutamine and 20 $\mathrm{U} \mathrm{ml}^{-1}$ interleukin-2 (IL-2). Human PBMCs were incubated with complete DMEM in gelatin flasks for $45 \mathrm{~min}$ at $37^{\circ} \mathrm{C}$ to collect monocytes. After detachment with $10 \mathrm{mM}$ EDTA, monocytes were washed with DMEM and resuspended in complete DMEM and plated in 48-well plates. Monocytes differentiated into macrophages during in vitro culture. Macaque monocytes from PBMCs were cultured in vitro for 7 days when the cells became macrophages as described. ${ }^{41} \mathrm{SIV}_{\text {mac251, }}, \mathrm{SIV}_{\text {mac239, }} \mathrm{SHIV}_{\mathrm{KU}-1}, \mathrm{HIV}-$ $1_{\mathrm{Bal}}$, and HIV-1 $1_{\mathrm{NL} 4-3}$ strains were obtained from the NIH AIDS Research Program. The R5 SHIV SF162P3N $_{\text {derived from the HIV-1 }}$ SF162 primary isolate ${ }^{42,43}$ was a generous gift from Dr. Cecilia ChengMayer (Aaron Diamond AIDS Research Center, New York, NY) and was propagated in phytohemagglutinin (PHA)-activated macaque PBMCs. The $50 \%$ tissue culture infectious doses $\left(\mathrm{TCID}_{50}\right)$ of SHIV $_{\text {SF162P3N }}$ was titrated in macaque PBMCs and the other virus stocks were titrated in TZM-bl cells. ${ }^{43}$ Briefly, PHA-activated PBMCs 

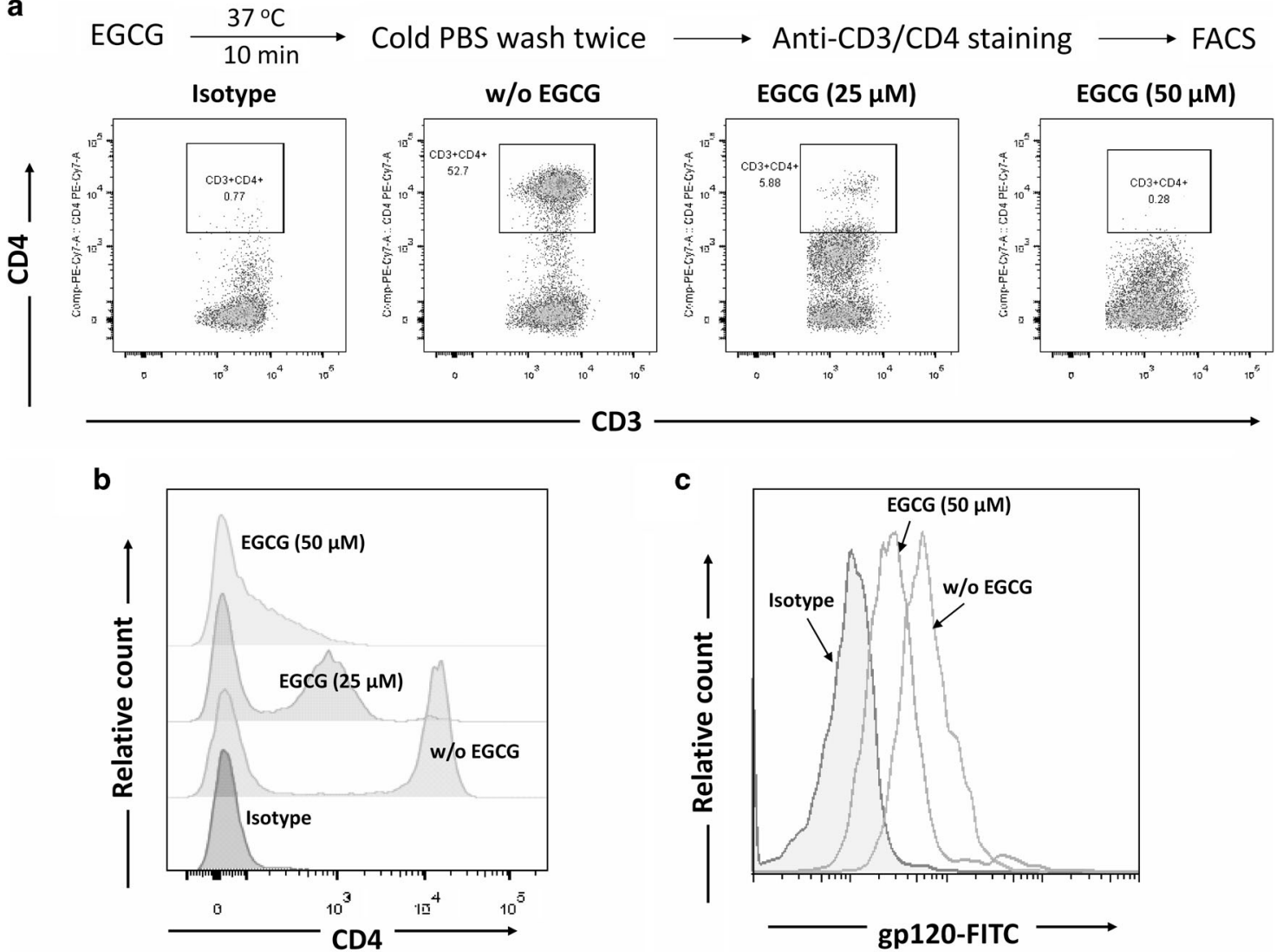

Fig. 5 Effect of EGCG on binding of anti-CD4 antibody and gp120 to CD4 T cells. a Peripheral blood mononuclear cells were incubated with indicated doses of EGCG for $10 \mathrm{~min}$. The reaction was then terminated with cold PBS. Cells were washed and then stained with anti-CD3 and anti-CD4 antibodies and subjected to flow cytometry analysis. The data shown are representative of three experiments with cells from three different donors. b Overlapping of the CD4 expression in PBMCs pretreated with indicated concentrations of EGCG. c Interference of binding of HIV-1 gp120 to CD4 ${ }^{+}$T cells. Purified CD4 ${ }^{+}$T cells were incubated with or without (w/o) $50 \mu \mathrm{M}$ of EGCG. The cells were then washed with PBS and further incubated with recombinant HIV-1 gp120 protein conjugated with FITC for $2 \mathrm{~h}$. The binding of gp120 to cells was indicated by intensity of FITC, which was examined by flow cytometry. Data are representative of three independent experiments

$\left(2.5 \times 10^{5}\right.$ per well) were infected with a serial dilution of the virus stock in quadruplicate, starting with a $1 / 5$ dilution in RPMI medium containing IL-2. The p27 antigens in culture supernatant were measured by SIV p27 ELISA (XpressBio, Frederick, MD). The $\mathrm{TCID}_{50}$ of the virus stocks were determined by the method of Reed and Muench. ${ }^{44}$

Rectal administration of EGCG and SHIV challenge

EGCG (Sigma-Aldrich, St. Louis, MO) was prepared as a stock solution $(20 \mathrm{mM})$ in PBS. The protective efficacy of EGCG on the rectal SHIV infection was evaluated in a repeated exposure macaque model using the SHIV $\mathrm{SF}_{162 \mathrm{P} 3 \mathrm{~N}}$, an extensively used SHIV strain in NHP model of sexual transmission. ${ }^{45,46}$ The animals were intramuscularly anesthetized with ketamine hydrochloride $(10 \mathrm{mg}$ $\left.\mathrm{kg}^{-1}\right)$ plus xylazine hydrochloride $\left(1 \mathrm{mg} \mathrm{kg}^{-1}\right)$. Anesthetized animals were maintained a ventral recumbent position and $2 \mathrm{ml}$ of EGCG $(5 \mathrm{mM})$ or PBS was administered atraumatically into the rectal vault prior to the intrarectal inoculation with SHIV $_{\text {SF162P3N }}$ at a dose of $10 \mathrm{TCID}_{50}{ }^{43}$ Animals that were not infected after the first inoculation were treated with EGCG or PBS and inoculated with SHIV again once biweekly. All the experiments were performed under highly controlled conditions by the same personnel using the same virus stock, inoculum dose and methods. Animals were considered infected and the virus challenges were stopped following two positive plasma SHIV RNA results. The experiments were stopped when all control animals became infected. ${ }^{9}$
Plasma SHIV RNA determination

Plasma SHIV RNA was analyzed by real-time RT-PCR for SIV/SHIV gag gene as previously described. ${ }^{9}$ Primer used were: forward $5^{\prime}$ TGGAGAACAAAGAAGGATGTCAAA-3', reverse $5^{\prime}$ - CACCAGATGACGCAGACAGTATTAT-3'. Probe sequence was 6FAMTTGGCACTAATGGAGCTAAGACCGAAAGTATT-BHQ1 (Gag-Btaq.2). Real-time PCR condition was: $95^{\circ} \mathrm{C}$ for $10 \mathrm{~min}, 40$ cycles of $95^{\circ} \mathrm{C}$ for $30 \mathrm{~s}$ and $60^{\circ} \mathrm{C}$ for $15 \mathrm{~s}$. Duplicate samples were analyzed and the limit of detection (LOD) was 200 SIV or SHIV RNA copies $\mathrm{ml}^{-1}$ plasma.

PCR amplification of proviral DNA and RNA

DNA and RNA was extracted from PBMCs, lymphocytes isolated from LNs and other GI tissues (Supplementary Table 3) with the AllPrep DNA/RNA/miRNA Universal Kit (Qiagen, Valencia, CA). Specimens were analyzed in duplicate with $200 \mathrm{ng}$ of DNA to amplify SHIV proviral DNA with the same primers and probes as for the plasma viral load assay described above. At week 20 after first SHIV challenge, inguinal LN and rectal mucosal were biopsied and subjected to RNA and proviral DNA detection. Intraepithelial lymphocytes from rectum or colon tissues were enriched to increase the sensitivity of the PCR as reported. ${ }^{9,47}$

gp120 binding assay

$\mathrm{CD}^{+} \mathrm{T}$ cells were incubated with $50 \mu \mathrm{M}$ of EGCG in RPMI- 1640 medium for $10 \mathrm{~min}$ at $37^{\circ} \mathrm{C}$. Large volume of cooled $\left(4^{\circ} \mathrm{C}\right) \mathrm{PBS}$ 


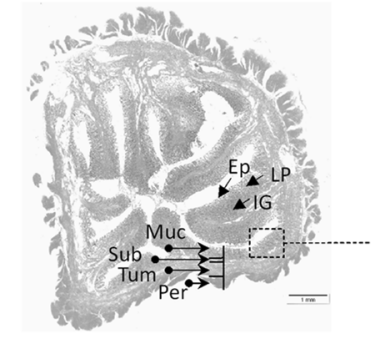

Rhesus macaque rectum microstructure Muc: mucosa $\longrightarrow$ Ep: epithelium Sub: submucosa $\longrightarrow$ LP: lamina propria Tum: Tunica muscularis IG: intestinal gland Per: Peritoneum
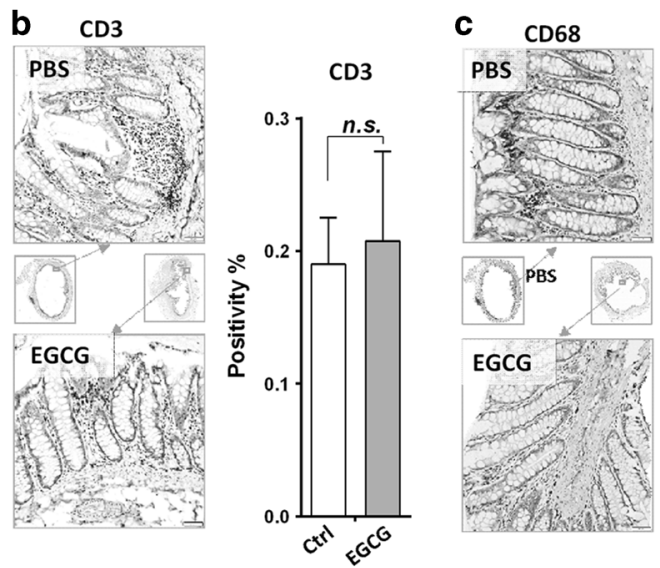

d
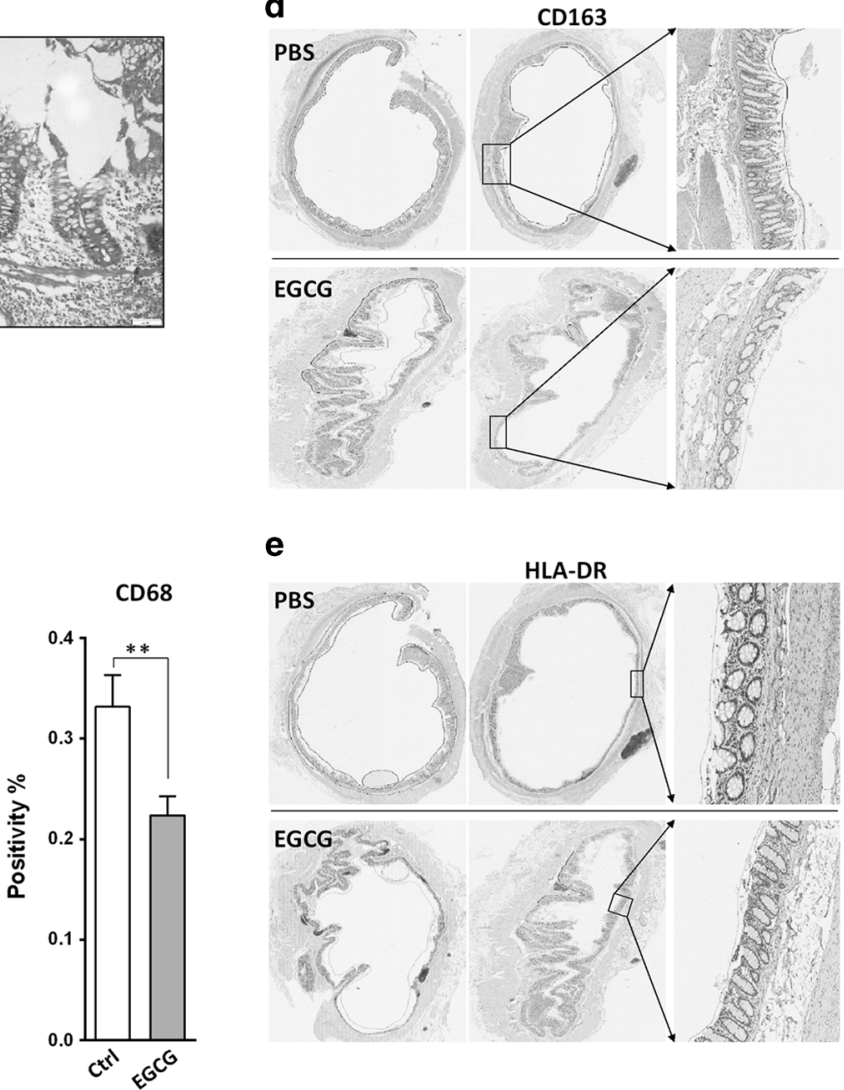

e

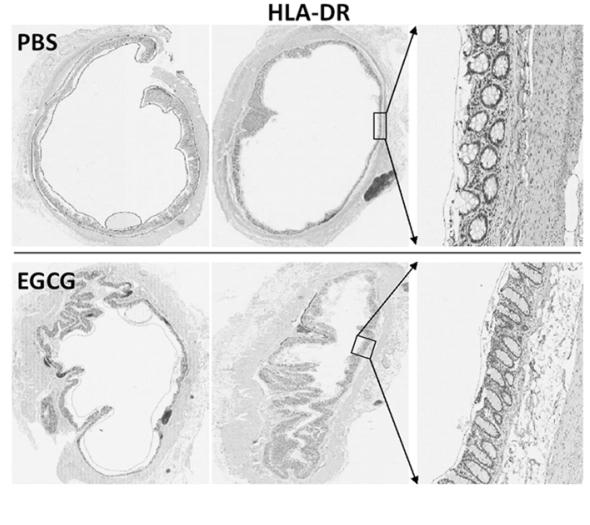

CD163

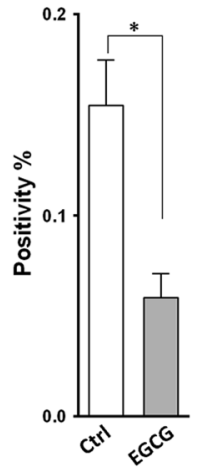

HLA-DR

Fig. 6 EGCG suppresses macrophage infiltration and immune activation in the rectal mucosa of SHIV-infected macaques. a Morphological observation of the microstructure of the rhesus macaque rectum. Architecture of the rectal mucosa of rhesus macaques as examined by hematoxylin and eosin staining. Magnification $\times 200$. b-e Immunohistochemistry staining of the cell infiltration and chronic immune activation in rhesus macaques intra-rectally treated with PBS or EGCG. EGCG $(5 \mathrm{mM}, 2 \mathrm{ml})$ or PBS ( 2 ml) was delivered to the rectum of rhesus macaques for $10 \mathrm{~min}$ prior to the challenge with SHIV $\mathrm{SF162P3N}\left(10 \mathrm{TCID}_{50}\right)$. Rectal tissues from two macaques were collected at autopsy $(96 \mathrm{~h}$ post-infection). Cell infiltrates were demonstrated by immunohistochemistry staining with anti-CD3 (b) or anti-CD68 (c) antibody. Tissue activation was examined by staining with anti-CD163 (d) or anti-HLA-DR (e) antibody. The positivity of mucosal tissue for CD3 ${ }^{+}$, CD68 $^{+}$, $\mathrm{CD}_{163^{+}}$, and $\mathrm{HLA}-\mathrm{DR}^{+}$cells in the rectum mucosa were quantified using the Aperio Image Scope software. The solid yellow lines in $\mathbf{d}$, e were used to designate regions, including the epithelium, lamina propria and intestinal glands of the rectal mucosa and submucosa, for algorithm analysis. Dotted yellow lines excluded the enclosed regions for the calculation. The positive cells within the mucosa and submucosa were counted per high-power field. At least two cross-sectioned rectal segments with different proximity to the anus were scanned and analyzed. Original magnification $\times 200$. Data are shown as mean \pm SD, which were analyzed using the two-tailed Student's $t$-test. ${ }^{*} P<0.05$ and ${ }^{* *} P<0.01$

was then added immediately after incubation to halt the interaction between cells and EGCG. Cells were washed twice with PBS and aliquoted for two sets of experiments. In the first set of experiment, the cells were stained directly with PBconjugated $\mathrm{mAb}$ against CD4 (Sk-3 clone) at room temperature subdue light for $45 \mathrm{~min}$. PB-conjugated mouse IgG of unrelated specificity was used as a negative control. The stained cells were then examined by flow cytometry for CD4 expression. In the second set of experiments, the EGCGtreated $\mathrm{CD}^{+}{ }^{+} \mathrm{T}$ cells were incubated with recombinant HIV gp120 protein conjugated with FITC $\left(20 \mathrm{\mu g} \mathrm{ml}^{-1}\right)$ for $1 \mathrm{~h}$. The cells were washed with PBS and the fluorescence intensity of gp120-FITC bound to the surface of $\mathrm{CD}^{+} \mathrm{T}$ cells was measured by the FACSCanto flow cytometer (BD Biosciences). Data were analyzed using the FlowJo data analysis software package (TreeStar Inc., USA).

\section{Serology}

Plasma SHIV-specific antibodies (IgG and IgM) of the study animals were measured by enzyme immunoassay (EIA) as instructed by the manufacturer (Genscreen ${ }^{\text {TM }}$ ULTRA HIV Ag-Ab; Bio-Rad).
Immunohistochemistry

Autopsied specimens from the rectal tissues were obtained 4 days after the rectal administration of PBS or EGCG and fixed in paraformaldehyde. The sections were incubated with mouse monoclonal antibodies against CD3 (F7.2.38) or CD68 (DK25; Dako; Carpinteria, CA), CD163 (EDHu-1; AbD Serotec; Raleigh, NC), HLADR (LN3; eBioscience; San Diego, CA) overnight at $4{ }^{\circ} \mathrm{C}$. Normal mouse serum was used as the negative antibody control for the specificity of the immunohistochemistry. The whole slides were digitally scanned using BX53 microscopy (Olympus, Japan) with ProScan III controller (Prior, UK). The tissue positivity for individual staining was assessed by Aperio ImageScope software (Leica Biosystems Inc., Buffalo Grove, IL).

\section{EGCG toxicity analysis}

The in vitro cytotoxicity of EGCG on the TZM-bl and PBMCs was determined by MTT assay or by flow cytometry using 7-amino actinomycin $D(7-A A D)$. The in vivo toxicity of EGCG on the rectal mucosa was examined by assessing the mucosal pathology, inflammatory response, stool consistency and bleeding. ${ }^{48,49}$ Briefly, EGCG was applied to rectum of the mice and the macaques. Fresh stools were then collected at $0,24,48$, and 72 
$h$ post EGCG administration for occult blood test. Stool consistency of mice were graded accordingly as previously described. $^{48}$ Inflammatory cytokines in the rectal tissues of the mice were measured by real time RT-PCR $72 \mathrm{~h}$ post-EGCG intrarectal administration. The impact of EGCG on rectal mucosal histology was analyzed by hematoxylin/eosin (HE) staining based on Obermeier's procedure. ${ }^{50}$ The rectum was divided into 3 equal segments with different proximity to the anus (proximal, middle, and distal), cross sectioned, and fixed in $10 \%$ formalin overnight. The tissues were then embedded in paraffin and serially sectioned at a thickness of $5 \mu \mathrm{m}$. Histological analysis was performed and evaluated in a blinded fashion. Each score represented the mean of nine sections.

Statistical analysis

The in vitro data were expressed as mean \pm standard deviation (SD) of triplicate cultures and statistical significance was assessed by Student's $t$ test. To protect the macaques from SHIV $\mathrm{SF}_{162 \mathrm{P} 3 \mathrm{~N}}$ infection was analyzed using Cox proportional hazard models based on the exact partial likelihood for discrete time. The number of challenges was used as a discrete time scale. The hazard ratios with $95 \%$ confidence intervals $(\mathrm{Cl})$ for the per-exposure relative reductions of acquisition risk were calculated for EGCG group as compared to the control group. Protection against acquisition of infection was also analyzed by log-rank (Mantel-Cox) tests. The statistical analyses of complete protection for the study animals against SHIV challenge were performed by Fisher's exact test. GraphPad Prism software (La Jolla, CA) was used for all statistical analyses. Significance was defined as ${ }^{*} P<0.05$, ${ }^{* *} P<0.01$, and ${ }^{* * *} P<0.001$.

\section{ACKNOWLEDGEMENTS}

This work was funded by the National Science and Technology Major Projects of Infectious Disease (2012ZX10004501-001-004), the Mega-Projects of Science Research for the 12th Five-Year Plan, China (2014ZX10001003005), the National Natural Sciences Foundation of China (81271334, 81201261, 81301428), the Fundamental Research Funds for the Central Universities (2042017kf0030 to J.B.L.) and in part by the National Institutes of Health Grants (DA041302 and DA022177 to W.Z.H.; DA040329 and MH109385 to J.L.L.). HIV/AIDS Research Award from the Robert Mapplethorpe Foundation (New York, NY) to J.L.L. is also acknowledged. We thank C.

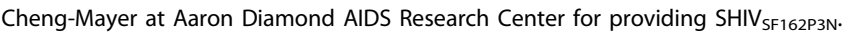

\section{AUTHOR CONTRIBUTIONS}

J.B.L., J.L.L., and W.Z.H. conceived of the study and experimental design and wrote the manuscript. J.B.L. and J.L.L. contributed equally to the work. J.B.L., J.L.L., H.L., Q.H. X., R.H.Z., and D.X.L. executed the macaque studies; X.W., K.Z., H.L., T.C.M., L.Z., and R. H.Z. performed viral loads and statistical analyses. M.Q.L. and W.Z. performed the SHIV antibody/plasma viral load measurement. J.B.L., J.L.L., and Q.H.X. performed IHC analyses and data analysis. All authors have read and approved the final version of this manuscript.

\section{ADDITIONAL INFORMATION}

The online version of this article (https://doi.org/10.1038/s41385-018-0025-4) contains supplementary material, which is available to authorized users.

Competing interests: The authors declare no competing interests.

\section{REFERENCES}

1. Ceballos, A. et al. Spermatozoa capture HIV-1 through heparan sulfate and efficiently transmit the virus to dendritic cells. J. Exp. Med. 206, 2717-2733 (2009).

2. Munch, J. et al. Semen-derived amyloid fibrils drastically enhance HIV infection. Cell 131, 1059-1071 (2007).

3. Usmani, S. M. et al. Direct visualization of HIV-enhancing endogenous amyloid fibrils in human semen. Nat. Commun. 5, 3508 (2014).

4. Zirafi, O. et al. Semen enhances HIV infectivity and impairs the antiviral efficacy of microbicides. Sci. Transl. Med. 6, 262ra157 (2014).
5. Cranage, M. et al. Prevention of SIV rectal transmission and priming of T cell responses in macaques after local pre-exposure application of tenofovir gel. PLoS Med. 5, e157 (2008).

6. Lederman, M. M. et al. Prevention of vaginal SHIV transmission in rhesus macaques through inhibition of CCR5. Science 306, 485-487 (2004).

7. Shattock, R. J. \& Rosenberg, Z. Microbicides: topical prevention against HIV. Cold Spring Harb. Perspect. Med. 2, a007385 (2012).

8. Veazey, R. S. et al. Protection of rhesus macaques from vaginal infection by vaginally delivered maraviroc, an inhibitor of HIV-1 entry via the CCR5 coreceptor. J. Infect. Dis. 202, 739-744 (2010).

9. Andrews, C. D. et al. Long-acting integrase inhibitor protects macaques from intrarectal simian/human immunodeficiency virus. Science 343, 1151-1154 (2014).

10. Andrews, C. D. et al. A long-acting integrase inhibitor protects female macaques from repeated high-dose intravaginal SHIV challenge. Sci. Transl. Med. 7, 270ra4 (2015).

11. Patton, D. L., Sweeney, Y. T. \& Paul, K. J. A summary of preclinical topical microbicide rectal safety and efficacy evaluations in a pigtailed macaque model. Sex. Transm. Dis. 36, 350-356 (2009).

12. Steinmann, J., Buer, J., Pietschmann, T. \& Steinmann, E. Anti-infective properties of epigallocatechin-3-gallate (EGCG), a component of green tea. Br. J. Pharmacol. 168, 1059-1073 (2013).

13. Nance, C. L., Siwak, E. B. \& Shearer, W. T. Preclinical development of the green tea catechin, epigallocatechin gallate, as an HIV-1 therapy. J. Allergy Clin. Immunol. 123, 459-465 (2009).

14. Castellano, L. M., Hammond, R. M., Holmes, V. M., Weissman, D. \& Shorter, J. Epigallocatechin-3-gallate rapidly remodels PAP85-120, SEM1(45-107), and SEM2 (49-107) seminal amyloid fibrils. Biol. Open 4, 1206-1212 (2015).

15. Hartjen, P. et al. Assessment of the range of the HIV-1 infectivity enhancing effect of individual human semen specimen and the range of inhibition by EGCG. AIDS Res. Ther. 9, 2 (2012).

16. Fassina, G. et al. Polyphenolic antioxidant (-)-epigallocatechin-3-gallate from green tea as a candidate anti-HIV agent. AIDS 16, 939-941 (2002).

17. Yamaguchi, K., Honda, M., Ikigai, H., Hara, Y. \& Shimamura, T. Inhibitory effects of (-)-epigallocatechin gallate on the life cycle of human immunodeficiency virus type 1 (HIV-1). Antivir. Res. 53, 19-34 (2002).

18. Li, S., Hattori, T. \& Kodama, E. N. Epigallocatechin gallate inhibits the HIV reverse transcription step. Antivir. Chem. Chemother. 21, 239-243 (2011).

19. Jiang, F. et al. The evaluation of catechins that contain a galloyl moiety as potential HIV-1 integrase inhibitors. Clin. Immunol. 137, 347-356 (2010).

20. Kawai, K. et al. Epigallocatechin gallate, the main component of tea polyphenol, binds to CD4 and interferes with gp120 binding. J. Allergy Clin. Immunol. 112, 951-957 (2003).

21. Williamson, M. P., McCormick, T. G., Nance, C. L. \& Shearer, W. T. Epigallocatechin gallate, the main polyphenol in green tea, binds to the T-cell receptor, CD4: potential for HIV-1 therapy. J. Allergy Clin. Immunol. 118, 1369-1374 (2006).

22. Zhou, R. H. et al. Epigallocatechin gallate inhibits macaque SEVI-mediated enhancement of SIV or SHIV infection. J. Acquir. Immune Defic. Syndr. 75, 232-240 (2017).

23. Nance, C. L. et al. Translational medicine in HIV-1 infection: preclinical and clinical development of the green tea catechin, Epigallocatechin Gallate, as therapy and immunological signatures. J. Allergy Clin. Immunol. 139, AB209 (2017).

24. Jenness, S. M. et al. Unprotected anal intercourse and sexually transmitted diseases in high-risk heterosexual women. Am. J. Public Health 101, 745-750 (2011).

25. Keele, B. F. \& Estes, J. D. Barriers to mucosal transmission of immunodeficiency viruses. Blood 118, 839-846 (2011).

26. Baggaley, R. F., White, R. G. \& Boily, M. C. HIV transmission risk through anal intercourse: systematic review, meta-analysis and implications for HIV prevention. Int. J. Epidemiol. 39, 1048-1063 (2010).

27. Hamza, A. \& Zhan, C. G. How can (-)-epigallocatechin gallate from green tea prevent HIV-1 infection? Mechanistic insights from computational modeling and the implication for rational design of anti-HIV-1 entry inhibitors. J. Phys. Chem. $B$ 110, 2910-2917 (2006).

28. Hauber, I., Hohenberg, H., Holstermann, B., Hunstein, W. \& Hauber, J. The main green tea polyphenol epigallocatechin-3-gallate counteracts semen-mediated enhancement of HIV infection. Proc. Natl Acad. Sci. USA 106, 9033-9038 (2009).

29. Roan, N. R. et al. The cationic properties of SEVI underlie its ability to enhance human immunodeficiency virus infection. J. Virol. 83, 73-80 (2009).

30. Roan, N. R. \& Munch, J. Improving preclinical models of HIV microbicide efficacy. Trends Microbiol. 23, 445-447 (2015).

31. Neurath, A. R., Strick, N. \& Li, Y. Y. Role of seminal plasma in the anti-HIV-1 activity of candidate microbicides. BMC Infect. Dis. 6, 150 (2006).

32. Chow, H. H. et al. Pharmacokinetics and safety of green tea polyphenols after multiple-dose administration of epigallocatechin gallate and polyphenon $E$ in healthy individuals. Clin. Cancer Res. 9, 3312-3319 (2003). 
33. Lambert, J. D. \& Yang, C. S. Mechanisms of cancer prevention by tea constituents. J. Nutr. 133, 3262S-3267S (2003).

34. Nguyen, M. M. et al. Randomized, double-blind, placebo-controlled trial of polyphenon $\mathrm{E}$ in prostate cancer patients before prostatectomy: evaluation of potential chemopreventive activities. Cancer Prev. Res. 5, 290-298 (2012).

35. Ullmann, U. et al. A single ascending dose study of epigallocatechin gallate in healthy volunteers. J. Int. Med. Res. 31, 88-101 (2003).

36. Yoon, A. J. et al. Topical application of green tea polyphenol (-)-Epigallocatechin3-gallate (EGCG) for prevention of recurrent oral neoplastic lesions. J. Orofac. Sci. 4, 43-50 (2012).

37. Lyseng-Williamson, K. A. \& Hoy, S. M. Polyphenon E $10 \%$ ointment: a guide to its use in the treatment of external genital and perianal warts. Drugs Ther. Perspect. 28, 6-9 (2012).

38. Stockfleth, E. et al. Topical Polyphenon $E$ in the treatment of external genital and perianal warts: a randomized controlled trial. Br. J. Dermatol. 158, 1329-1338 (2008).

39. Stalmach, A., Troufflard, S., Serafini, M. \& Crozier, A. Absorption, metabolism and excretion of Choladi green tea flavan-3-ols by humans. Mol. Nutr. Food Res. 53, S44-S53 (2009).

40. Zhou, Q. et al. Investigating the stability of EGCg in aqueous media. Curr. Sep. 20, 83-86 (2003).

41. Sang, M., Liu, J. B., Dai, M., Wu, J. G. \& Ho, W. Z. Toll-like receptor 3 signaling inhibits simian immunodeficiency virus replication in macrophages from rhesus macaques. Antivir. Res. 112, 103-112 (2014).
42. Shakirzyanova, M. et al. Pathogenic consequences of vaginal infection with CCR5tropic simian-human immunodeficiency virus SHIVSF162P3N. J. Virol. 86, 9432-9442 (2012)

43. Harouse, J. M., Gettie, A., Tan, R. C., Blanchard, J. \& Cheng-Mayer, C. Distinct pathogenic sequela in rhesus macaques infected with CCR5 or CXCR4 utilizing SHIVs. Science 284, 816-819 (1999).

44. Reed, L. J. \& Muench, H. A simple method of estimating fifty percent endpoints. Am. J. Hyg. 27, 493-497 (1938).

45. Ren, W. et al. Mucosal transmissibility, disease induction and coreceptor switching of R5 SHIVSF162P3N molecular clones in rhesus macaques. Retrovirology 10, 9 (2013).

46. Tsai, L., Tasovski, I., Leda, A. R., Chin, M. P. \& Cheng-Mayer, C. The number and genetic relatedness of transmitted/founder virus impact clinical outcome in vaginal R5 SHIVSF162P3N infection. Retrovirology 11, 22 (2014).

47. Mehandru, S. et al. Primary HIV-1 infection is associated with preferential depletion of CD4 + T lymphocytes from effector sites in the gastrointestinal tract. J. Exp. Med. 200, 761-770 (2004)

48. Kim, J. J., Shajib, M. S., Manocha, M. M. \& Khan, W. I. Investigating intestinal inflammation in DSS-induced model of IBD. J. Vis. Exp. e3678 (2012).

49. Tabet, S. R. et al. Safety and toxicity of nonoxynol-9 gel as a rectal microbicide. Sex. Transm. Dis. 26, 564-571 (1999).

50. Obermeier, F. et al. Interferon-gamma (IFN-gamma)- and tumour necrosis factor (TNF)-induced nitric oxide as toxic effector molecule in chronic dextran sulphate sodium (DSS)-induced colitis in mice. Clin. Exp. Immunol. 116, 238-245 (1999). 